\title{
Tailoring Project-Based Learning for a Communications Engineering Course
}

\author{
José A. López-Salcedo, Gonzalo Seco-Granados \\ Signal Processing for Communications and Navigation (SPCOMNAV), \\ Department of Telecommunications and Systems Engineering, Universitat Autònoma de Barcelona (UAB) \\ Engineering School, Campus Universitari s/n, 08193 Bellaterra (Barcelona), Spain. \\ Email: \{jose.salcedo, gonzalo.seco\}@uab.es
}

\begin{abstract}
This paper describes the introduction of a ProblemBased Learning (PBL) methodology within an undergraduate course of Telecommunication Engineering at the Universitat Autònoma de Barcelona (UAB). The PBL approach is in line with the convergence towards the common European Higher Education Area (EHEA), in which students will play a prominent and active role in their own learning. The selected course for this experience is related with the Design of Advanced Digital Receivers, a topic where real-life engineers have often to cope with ill structured, ambiguous and complex problems for which more than one correct answer may be possible. The results to be presented herein are based on the instructors' experience gained after two consecutive years of PBL practice on this course, and also on the feedback obtained from students.
\end{abstract}

\section{INTRODUCTION}

The adoption of Problem-Based Learning (PBL) methodologies is one of the common approaches adopted by many European universities in the redefinition of current Engineering courses to the new framework of the European Higher Education Area (EHEA). In this new framework, instructors will move from their traditional role of "stand and deliver" to a more dynamic and student-centered role of learning facilitator [1]. The motivation of this new role is to guide students by helping them:

- to explore the richness of open-ended questions,

- to develop their own critical thinking and search for meaning,

- to raise issues that need to be considered,

- to monitor progress,

- to potentiate their self-learning skills,

- to create and maintain a warm atmosphere for stimulating individuals to share experiences and ideas.

In summary, the instructor's new role will be based on guiding and helping students rather than on being a source of information with answers to all of their questions. A remarkable aspect is that the student's reflection becomes a key part of the PBL methodology, since reflection develops professional skills [2], problem solving capabilities [3] and improves lifetime learning [4], thus providing many of the required attributes for a successful professional working life.

\section{CASE OF STUDY}

In this context of student-oriented learning, this paper describes the experience of a last-year Engineering course where a combination of both traditional and PBL methodologies have been implemented to follow the EHEA. The course is entitled "Design of Advanced Digital Receivers" and it is an optional course within the Msc. in Telecommunication Engineering at the Universitat Autònoma de Barcelona (UAB). The course was recently approved by the University, with the first lectures being held on the Autumn semester of 2007. The course work involves 6 conventional credits (i.e. no ECTS credits, but 10 hours/credit) which are distributed in 3 credits for theoretical lectures, 1.5 credits for directed problem solving and 1.5 credits for laboratory sessions. Although the Msc. program has not been adapted yet to EHEA, this specific course was decided to be launched by taking into consideration the EHEA philosophy.

The motivation of the faculty members in charge of this course was to offer a highly technical preparation for undergraduate students in a specific field where highly skilled professionals are being sought by industry. To do so, the faculty members performed a previous work on translating the demands from industry into a series of competencies [5], both specific and transversal (analysis of complex solutions, management of uncertainty and incomplete data, team work, public speaking, capacity of synthesis, global view, discussion under stress, etc.). After a thorough benefits-costs analysis, the course was decided to be implemented in such a way that $75 \%$ of the credits were dedicated to "conventional methodologies" and $25 \%$ of the credits where dedicated to PBL. PBL seemed to be a good choice for the design of this course since PBL focuses on addressing typically ill-structured problems with many possible solutions, a very similar working scenario to that encountered in real-life engineering problems [6], [7], [8].

The first $75 \%$ of traditional learning was divided into $55 \%$ for lectures and $20 \%$ for directed problem solving and laboratory. This $20 \%$ of practical learning under the traditional methodology allows students to face the concepts presented in class in a controlled environment where the initial conditions and the problem statement are clearly presented. The goal here is to consolidate the technical concepts presented in class and 
explore the application to particular test cases. That is to say, while the first part of lectures would correspond to the first phase of learning (i.e. the acquisition phase) the second part of directed problem solving would correspond to the learning stages of fluency and generalization [9]. However, some of the identified competencies were found not to be properly covered by this approach, especially the ones related with facing multiple answer problems, dealing with uncertainty, stress and incomplete data, or being able to adapt to new situations. In that case, PBL seemed to provide the answer for complementing the lacks of traditional learning. Nevertheless, implementing the whole course based on the PBL philosophy was discarded due to the large amount and complexity of the technical contents. It is important to remark that one of the very first intentions of the course was to keep a high technical level while providing the students with new concepts. For this purpose, a minimum fixed amount of lectures deemed to be satisfactory. Achieving the same results with a purely PBL-based course would have posed significant obstacles for students to complete the required knowledge acquisition phase within the time planning of the Msc. program.

\section{PBL Methodology and Role Playing}

For the $25 \%$ of the course devoted to PBL, the intention was to reflect a real-life situation that students could face in their professional career. This situation was decided to be the standardization process of a given technology. To do so, instructors were assumed to be part of the committee that had to decide the standardization of a given technological solution among two possible candidates. Students were organized into two groups, and each of these groups was responsible of convincing the committee to adopt their solution. This working scenario also introduced the students into the real-life problems to be faced by standardization bodies, the motivations that move industry to go for standardization of their products and technical innovations, and to familiarize students with the fact that no unique nor optimal solution can often be found in practice. When discussing each of the candidate technologies with the committee, the groups should also learn to defend their option even though they may know in advance that it is inferior to that of the competing group. In practice, similar situations may occur when commercial or economic reasons prevail over technical excellence.

\section{A. Organization of the PBL activities}

This section briefly describes the overall organization of the PBL part of the course under study. This structure is common to both the 2007 and 2008 academic years where the course has been held. The following activities were carried out during the 3 weeks in which the PBL experience took place:

1) Day-1: Presentation of the PBL activity. This is a 2 hours class where the problem (i.e. the technology to standardize) is introduced to the students. The following topics are covered:

- Objectives of the proposed activity.

- Problem statement.
- Presentation of the two candidate solutions.

- Brief review of pros and cons of both solutions.

- Suggestion of some key issues to be analyzed.

- Form two groups of students (the total number of students attending the course allowed two groups with 5 students each).

- Provide the agenda for the PBL activities to follow.

- Agreement on the evaluation method.

2) Day-2: Theoretical class of 1 hour duration where some basic concepts are refreshed, or where some new concepts or algorithms are introduced. The goal of this session is to orient the students and reduce their initial uncertainty. Keeping the students' uncertainty down to an acceptable level is an important aspect for maximizing their performance, as suggested by the inverted-U relationship between performance and stress [10].

3) The groups are lead to work on their own for a period of one week.

4) Day-3: Progress meeting of 1 hour duration with each of the groups, separately. The motivation of these disjoint meetings is not to mix both groups within the same class. By doing so, the arguments of each group can be kept confidential as well as their technical doubts and strategies to convince the committee. It is important to recall that both groups are indeed competing for their solution to be accepted, and thus preserving confidentiality is an important part of the story.

5) The groups are lead to work on their own for three days. During this period they will concentrate on consolidating their technical arguments and preparing the final debate.

6) Day-4: The final debate takes place.

- Each group has 20-30 minutes for presenting their solution and convincing the committee.

- When both groups have presented their proposal, a 20 minutes debate between both groups is initiated and moderated by the instructor.

- During both the group presentation and the final debate, the instructor is evaluating the individual contribution of each of the students. After the final debate, the instructor provides some constructive criticism by highlighting the weak points of the presentations, and praises the students achievements.

From the above description, it can be seen that the overall PBL process involves two short technical meetings of 1 hour duration each, with the aim of monitoring and orienting the work of students. Certainly, just one meeting would not be enough to provide the students with some basic keys and concepts to exploit their self-learning. On the other hand, performing more than two meetings would transmit the feeling to students that "there is no need to worry at all since the instructor will end up by giving us the solution to the problem". Finally, it should also be mentioned that the final debate is carried out in a meeting room, not in a class, in order to create a more realistic environment to the one typically encountered in practice. 


\section{B. Course Evaluation}

The two different pedagogical methodologies that were introduced during the course are equally weighted to form the final mark. That is, $50 \%$ of this mark corresponds to the learning acquired through traditional methodology with lectures, directed problem solving and laboratory exercises. The other $50 \%$ of the mark comes from the evaluation of the PBL activities. In that case, both the instructor and the students were involved in the evaluation process. The instructor's contribution to the PBL marks consisted in the assessment of the students learning process and the evaluation of the students skills in defending their candidate solution during the final debate. As for the students' contribution, they were required to be involved in a peer assessment protocol: each student had to evaluate the rest of students in their group [11].

Such a peer assessment protocol has been reported in the literature to reinforce the acquisition of self-directed learning skills [12]. Critical skills and metacognitive skills are also improved due to the enrollment of students in their own assessment. However, some possible drawbacks may be observed such as the existence of bias in peer marking due to the interpersonal relationships between students within a given group [13]. This effect is also noticed by some students, who often perceive peer assessment as unreliable and unfair [14]. In the course under study the faculty members agreed that testing a peer assessment experience would be interesting. However, for this assessment to succeed, the possible drawbacks or unfairness perceived by the students should be minimized. To do so, two groups of students were formed by carefully selecting the members in order to avoid possible interpersonal relationships ${ }^{1}$. The a-priori knowledge that the instructors had for most of the attending students was combined with the Basadur problem solving profile for assigning individuals into each of their teams [10]. The Basadur profile states that for a team to be effective in problem solving, it must have strengths and interests in all four quadrants of the model: "action taking", "problem finding", "decision making" and "idea finding".

Once the groups were formed, the peer assessment protocol was presented. The procedure is as follows:

Each student has to evaluate (in a confidential manner) the members of his/her group, except for himself. If the total number of group members is $N$ students, each student within a group has $7(N-1)$ points to distribute among the remaining $N-1$ students. In an impartial distribution of points, it is possible that a given student would decide to give 7 points to each of his/her colleagues. If this occurs, peer assessment would have to be discarded since no useful insights are provided to the instructor for discriminating between lazy and productive students. However, the results obtained for this method show that for the case under study, there is a

\footnotetext{
${ }^{1}$ It should be mentioned that since this course is a last-year optional course, the enrolled students were all familiar to the instructors, who met them in previous undergraduate courses. This a-priori information helped in preventing closely related students to fall within the same group.
}

surprisingly close match between the students peer assessment and the instructor's own evaluation.

\section{EVAluation of The PBL Experience}

Two different results are to be reported in this section: the first one is concerned with the students personal evaluation of the course (i.e. their satisfaction), and the second one is concerned with the reliability analysis of the students peer assessment.

\section{A. Students' Feedback}

At the end of the course, a questionnaire was given to the students with the aim of reflecting their degree of satisfaction with the whole course, and with the PBL activities in particular. A set of 10 questions were provided, as listed below. The questions had to be scored from 0 (complete disagreement) to 10 (complete agreement), except for questions Q3 and Q4 that required a written explanation from the student.

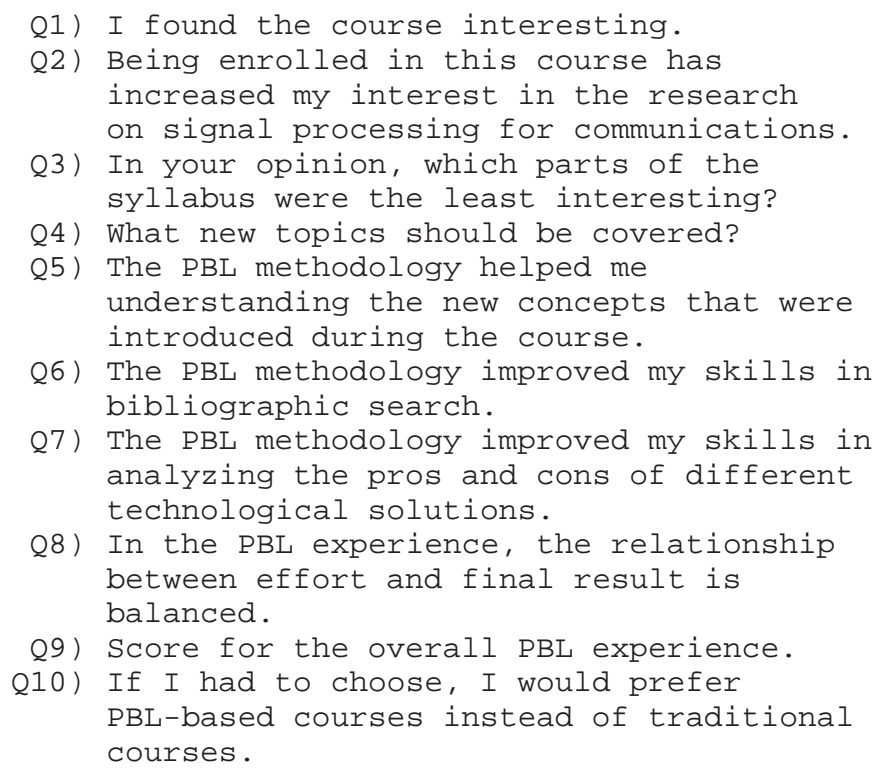

The results for the questions listed above are shown in Fig. 1 for the two academic years when the course has been held. The two most straightforward conclusions that can be drawn from these results are the following:

- Students show a very high level of satisfaction with the overall course. This is reflected in a mean score of 8.35 for Q1 in 2007, and 9.1 in 2008. There is also a high degree of agreement among students for this satisfaction. This can be observed in Fig. 2 where the scores for Q1 are found to exhibit a rather low dispersion.

- Students seem to be reluctant to be enrolled in purely PBL based courses. This can be observed in the poor results for Q10, especially for the year 2008.

At first glance, these conclusions suggest that a combination of both traditional and PBL methodologies is indeed a good approach for applying self-learning strategies into an engineering course. This is true not only from the students point of view 


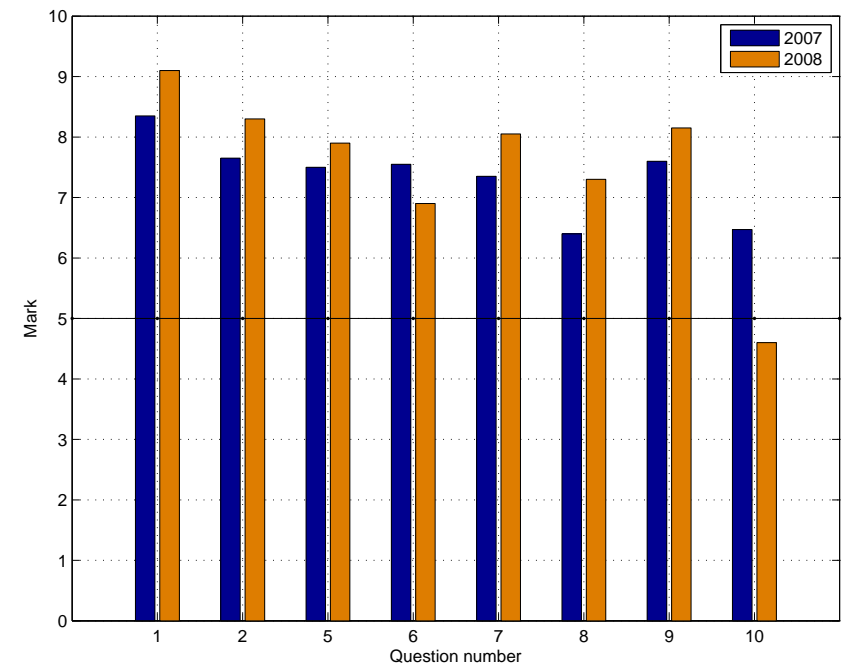

Fig. 1. Mean scores given by the students to questions related with the PBL experience for the two years in which the course was held.

(which is confirmed in the scores), but also from the instructors point of view, which do often argue that advanced courses in Engineering studies are difficult to be purely translated to PBL while maintaining the same levels of quality and technical skills. In that sense, our experiment seems to confirm that the experience of implementing a combination of both traditional and PBL methodologies is a good choice that could be applied to other engineering courses.

On the opposite side, it is important to remark the poor scores assigned to some of the questions, especially to Q10. This question was asking students about the convenience of attending purely PBL-based courses, and their answers did not clearly support that idea (especially in the results for 2008). Two possible causes have been identified for explaining this reluctance of students to be enrolled in purely PBL courses. First of all, the fact that the Msc. program has not been adapted to EHEA yet. Since this course under study is a last-year course, students are somehow used to the traditional learning methodologies of their previous courses. This reluctance of students to change their traditional perspective of the educational procedure has been reported in the literature as one of the obstacles in introducing PBL [15]. Such a resistance suggests that the benefit-cost tradeoff incurred by PBL-based activities is often not perceived to be advantageous from the student point of view. This additional effort that students have to dedicate to reflect, investigate, monitor and interact in selflearning groups, has to be taken into consideration by the instructors when dimensioning the whole course. Otherwise, PBL will not be effective and students will be discouraged to be enrolled in the course.

\section{B. Results of the Peer-Assessment Protocol}

The peer-assessment protocol described in Section III-B was carried out at the end of the final debate session. Students were required to evaluate their colleagues' work during the PBL part of the course, especially the work carried out during the

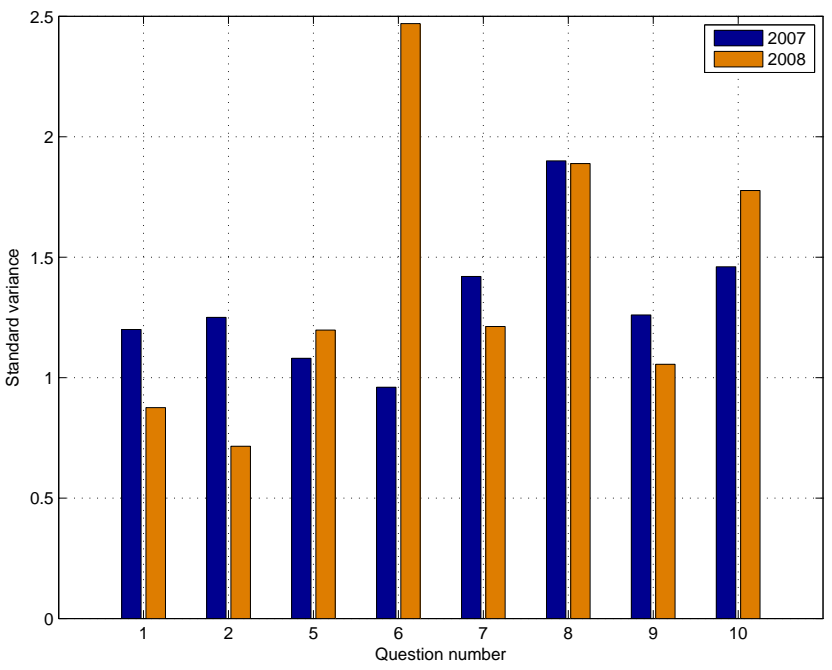

Fig. 2. Standard deviation for the scores given by the students to questions related with the PBL experience for the two years in which the course was held.

self-learning period and internal group meetings. For the peerassessment, students were requested to think about the degree of involvement of their colleagues, their effort in achieving the common goal, their leadership skills, the work planning and organization, and to reflect all these aspects into a single score ranging from 0 to 10 . These were the same criteria that instructors were also using for drawing their own evaluation for each of the students.

Later on, both scores were compared and analyzed with the aim of assessing the reliability that students self-evaluation could have in the determination of the final marks. This comparison is shown in Fig. 3, where each point is representing the PBL evaluation of a given student. The $\mathrm{X}$-axis represents the mean score obtained from the students peer-assessment and the Y-axis represents the score obtained according to the instructor's evaluation. To further highlight the relationship between both scores, a straight line can easily be traced in this figure for representing the linear regression for the available data. The slope of this line is 1.33 , thus showing that students tend to underestimate their colleagues' work. Probably this can be interpreted as a countermeasure to uncertainty, since peer-assessment is confidential. In any case, this deviation is not significant at all and still preserves the close match between the students' evaluation and the instructors' opinions. This close match can also be observed when calculating the correlation between the two sources of data. In terms of the $\mathrm{R}$-squared parameter, this experiment achieves $R^{2}=0.8148$, a value close to 1 that suggests again the close match with the instructors evaluation, and it confirms the validity of the proposed peer assessment protocol.

\section{Lessons Learned}

Based on the observations made by the instructors all along the course, and based on the comments provided by students at the end of the PBL experience, the following lessons have 


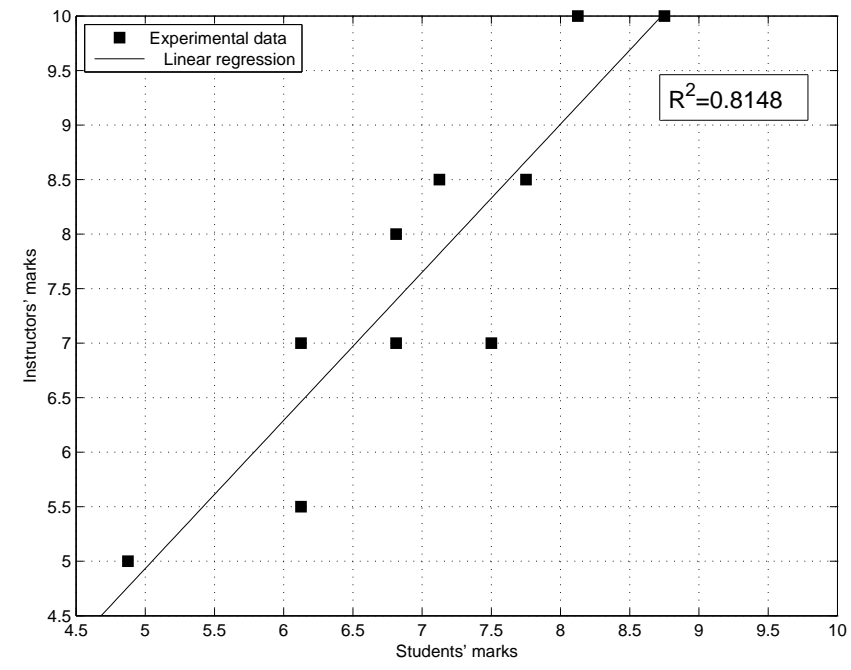

Fig. 3. Scatter plot for the peer assessment. The X-axis corresponds to the mean marks from the students peer assessment. The Y-axis corresponds to the marks independently assigned by the instructors. The solid line represents the linear regression.

\section{been learned:}

- The balance between available time and required effort has to be carefully analyzed before setting up any PBL activity. PBL should allow students to bring out their very best, and not to become a stressful load. For this reason, and based on the feedback from students and the course progress, subsequent editions of this course should allocate more time to the PBL part. A more reasonable distribution of traditional learning vs PBL activities would be on the order of $65-70 \%$ vs $35-30 \%$, instead of the current distribution of $75 \%$ vs $25 \%$.

- Students become motivated when representing real-life roles (e.g. as in the standardization committee adopted in this course). This situation wakes up their curiosity and interest on the topics to be developed.

- Students become even more motivated when competition is established between groups [16].

- When defining the objectives of the PBL role playing, students have to be reminded that the arguments for convincing the evaluating committee are based on quality rather than on quantity.

- All groups should know the candidate technologies that their competitors will be defending. This allows students to have in mind all the alternative solutions, elaborate on their pros and cons, and thus consolidate a more solid position for the argumentation of their own technology in the final debate.

- Feedback must be provided after completing the PBL role playing activities, as a part of the overall evaluation process.

\section{Conclusions}

This paper presented an example of the application of PBL methodologies to an engineering course on Design of
Advanced Digital Receivers. This is a course that combines both traditional lectures and directed problem solving classes, with dynamic self-learning strategies based on PBL. The results show that this experience is very successful, and that the combination of both approaches allows students to acquire advanced technical skills as well as transversal competencies demanded by industry and society to new graduates. This way of organizing the course has been exciting and motivating for both instructors and students, since as the course was progressing, there was an increasing feeling that classes were really useful and that contents were being assimilated. The good feedback received from students confirms this statement (as reflected in Q1 of the feedback questionnaire), and shows that this type of experience where traditional and PBL methodologies are combined can be a good choice for other engineering courses.

\section{REFERENCES}

[1] D. R. Woods, Problem-Based Learning: How to Gain the Most from PBL. Waterdown Illustrated, 1996.

[2] D. Schon, Educating the Reflective Practitioner: Toward a New Design for Teaching and Learning in the Professions. San Francisco: JosseyBass, 1987.

[3] R. Kimbell, K. Stables, T. Wheeler, A. Wosniak, and V. Kelly, The Assessment of Performance in Design and Technology. London: National Examination Assessment Council, Newcombe House, 1991.

[4] H. G. Scmidt, "Problem-based learning: rationale and description," Medical Education, vol. 17, pp. 11-16, 1983.

[5] J. Armarego, "Beyond PBL: preparing graduates for professional practice," in Proc. 20th Conference on Software Engineering Education and Training (CSEET), 2007.

[6] K. C. Bower, T. W. Mays, and C. M. Miller, "Small group, selfdirected problem based learning development in a traditional engineering problem," in Proc. ASEE/IEEE Conference on Frontiers in Education, 2004, pp. 16-21.

[7] A. P. Massey, V. Ramesh, and V. Khatri, "Design, development and assessment of mobile applications: the case for problem-based learning," IEEE Transactions on Education, vol. 49, no. 2, pp. 183-192, 2006.

[8] J. Perrenet, S. Bouhuijs, and J. Smits, "The suitability of problem based learning for engineering education," Teaching in Higher Education, vol. 5, no. 3, pp. 345-358, 2000.

[9] N. G. Haring, T. C. Lovitt, M. D. Eaton, and C. L. Hansen, The Fourth R: Research in the Classroom. Merrill Publishing Co., 1978.

[10] T. Peterson, "So you're thinking of trying problem based learning? Three critical success factors for implementation," Journal of Management Education, vol. 28, pp. 630-648, 2004.

[11] T. Papinczak, L. Young, and M. Groves, "Peer assessment in problembased learning: a qualitative study," Advances in Health Sciences Education, vol. 12, no. 2, pp. 169-186, May 2006.

[12] R. Ballantyne, K. Hughes, and A. Mylonas, "Developing procedures for implementing peer assessment in large classes using an action research process," Assessment and Evaluation in Higher Education, vol. 27, no. 5, pp. 427-441, 2002.

[13] D. M. Sluijmans, G. Moerkerke, J. J. van Merrienboer, and F. J. Dochy, "Peer assessment in problem-based learning," Studies in Educational Evaluation, vol. 27, no. 2, pp. 153-173, 2001.

[14] L. McDowell, "The impact of innovative assessment on student learning," Innovation in Education and Training International, vol. 32, no. 4, pp. 302-313, 1995.

[15] R. Littlefield, "Introducing problem-based learning to graduate students: an autoethnographic account of risk-taking," in Proc. International Communication Association, Networking Communication Research, Dresden, Germany, June 19-23 2006.

[16] C. C. Bonwell and J. A. Eison, Active Learning: Creating Excitement in the Classroom. The George Washington University, 1991. 